\title{
FORMATION OF VERSIONS OF SOME DYNAMIC INEQUALITIES UNIFIED ON TIME SCALE CALCULUS
}

\author{
Muhammad Jibril Shahab Sahir \\ Department of Mathematics, University of Sargodha, Sub-Campus Bhakkar, \\ Pakistan \& GHSS, 67/ML, Bhakkar, Pakistan \\ jibrielshahab@gmail.com
}

\begin{abstract}
The aim of this paper is to present some comprehensive and extended versions of classical inequalities such as Radon's Inequality, Bergström's Inequality, the weighted power mean inequality, Schlömilch's Inequality and Nesbitt's Inequality on time scale calculus. In time scale calculus, results are unified and extended. The theory of time scale calculus is applied to unify discrete and continuous analysis and to combine them in one comprehensive form. This hybrid theory is also widely applied on dynamic inequalities. The study of dynamic inequalities has received a lot of attention in the literature and has become a major field in pure and applied mathematics.
\end{abstract}

Keywords: Radon's Inequality, Bergström's Inequality, the weighted power mean inequality, Schlömilch's Inequality, Nesbitt's Inequality.

\section{Introduction}

The time scale calculus has a scope for many applications in the field of dynamic inequalities. The time scale calculus was initiated by Stefan Hilger as given in [11]. A time scale is an arbitrary nonempty closed subset of the real numbers. The time scale calculus is studied as delta calculus, nabla calculus and diamond- $\alpha$ calculus. Basic work on dynamic inequalities is done by Ravi Agarwal, George Anastassiou, Martin Bohner, Allan Peterson, Donal O'Regan, Samir Saker and many other authors.

We will prove the following results given in (1.1), (1.2) and (1.3) on time scales.

The inequality from (1.1) is called Bergström's Inequality as given in [4-6, 13].

Theorem 1. If $n \in \mathbb{N}, x_{k} \in \mathbb{R}$ and $y_{k}>0, k \in\{1,2, \ldots, n\}$, then

$$
\frac{\left(\sum_{k=1}^{n} x_{k}\right)^{2}}{\sum_{k=1}^{n} y_{k}} \leq \sum_{k=1}^{n} \frac{x_{k}^{2}}{y_{k}}
$$

with equality if and only if

$$
\frac{x_{1}}{y_{1}}=\frac{x_{2}}{y_{2}}=\ldots=\frac{x_{n}}{y_{n}}
$$

The upcoming result is called Radon's Inequality as given in [15]. 
Theorem 2. If $n \in \mathbb{N}, x_{k} \geq 0$ and $y_{k}>0, k \in\{1,2, \ldots, n\}$ and $\beta \geq 0$, then

$$
\frac{\left(\sum_{k=1}^{n} x_{k}\right)^{\beta+1}}{\left(\sum_{k=1}^{n} y_{k}\right)^{\beta}} \leq \sum_{k=1}^{n} \frac{x_{k}^{\beta+1}}{y_{k}^{\beta}} .
$$

Inequality (1.2) is widely studied by many authors because it has many applications.

The following inequality is generalized Radon's Inequality as given in [10].

Theorem 3. If $n \in \mathbb{N}, x_{k} \geq 0, y_{k}>0, k \in\{1,2, \ldots, n\}$ and $\beta \geq \gamma \geq 0$, then

$$
n^{\gamma-\beta} \frac{\left(\sum_{k=1}^{n} x_{k}\right)^{\beta+1}}{\left(\sum_{k=1}^{n} y_{k}\right)^{\gamma}} \leq \sum_{k=1}^{n} \frac{x_{k}^{\beta+1}}{y_{k}^{\gamma}}
$$

with equality if and only if $x_{1}=x_{2}=\ldots=x_{n}$ and $y_{1}=y_{2}=\ldots=y_{n}$.

In this paper, it is assumed that all considerable integrals exist and are finite and $\mathbb{T}$ is a time scale, $a, b \in \mathbb{T}$ with $a<b$ and an interval $[a, b]_{\mathbb{T}}$ means the intersection of a real interval with the given time scale.

\section{Preliminaries}

We need here basic concepts of delta calculus. The results of delta calculus are adapted from monographs $[7,8]$.

For $t \in \mathbb{T}$, the forward jump operator $\sigma: \mathbb{T} \rightarrow \mathbb{T}$ is defined by

$$
\sigma(t):=\inf \{s \in \mathbb{T}: s>t\} .
$$

The mapping $\mu: \mathbb{T} \rightarrow \mathbb{R}_{0}^{+}=[0,+\infty)$ such that $\mu(t):=\sigma(t)-t$ is called the forward graininess function. The backward jump operator $\rho: \mathbb{T} \rightarrow \mathbb{T}$ is defined by

$$
\rho(t):=\sup \{s \in \mathbb{T}: s<t\} .
$$

The mapping $\nu: \mathbb{T} \rightarrow \mathbb{R}_{0}^{+}=[0,+\infty)$ such that $\nu(t):=t-\rho(t)$ is called the backward graininess function. If $\sigma(t)>t$, we say that $t$ is right-scattered, while if $\rho(t)<t$, we say that $t$ is left-scattered. Also, if $t<\sup \mathbb{T}$ and $\sigma(t)=t$, then $t$ is called right-dense, and if $t>\inf \mathbb{T}$ and $\rho(t)=t$, then $t$ is called left-dense. If $\mathbb{T}$ has a left-scattered maximum $M$, then $\mathbb{T}^{k}=\mathbb{T}-\{M\}$, otherwise $\mathbb{T}^{k}=\mathbb{T}$.

For a function $f: \mathbb{T} \rightarrow \mathbb{R}$, the delta derivative $f^{\Delta}$ is defined as follows:

Let $t \in \mathbb{T}^{k}$, if there exists $f^{\Delta}(t) \in \mathbb{R}$ such that for all $\epsilon>0$, there exists a neighborhood $U$ of $t$, such that

$$
\left|f(\sigma(t))-f(s)-f^{\Delta}(t)(\sigma(t)-s)\right| \leq \epsilon|\sigma(t)-s|,
$$

for all $s \in U$, then $f$ is said to be delta differentiable at $t$, and $f^{\Delta}(t)$ is called the delta derivative of $f$ at $t$.

A function $f: \mathbb{T} \rightarrow \mathbb{R}$ is said to be right-dense continuous (rd-continuous), if it is continuous at each right-dense point and there exists a finite left limit at every left-dense point. The set of all rd-continuous functions is denoted by $C_{r d}(\mathbb{T}, \mathbb{R})$.

The next definition is given in $[7,8]$. 
Definition 1. A function $F: \mathbb{T} \rightarrow \mathbb{R}$ is called a delta antiderivative of $f: \mathbb{T} \rightarrow \mathbb{R}$, provided that $F^{\Delta}(t)=f(t)$ holds for all $t \in \mathbb{T}^{k}$. Then the delta integral of $f$ is defined by

$$
\int_{a}^{b} f(t) \Delta t=F(b)-F(a)
$$

The following results of nabla calculus are taken from $[3,7,8]$.

If $\mathbb{T}$ has a right-scattered minimum $m$, then $\mathbb{T}_{k}=\mathbb{T}-\{m\}$, otherwise $\mathbb{T}_{k}=\mathbb{T}$. A function $f: \mathbb{T}_{k} \rightarrow \mathbb{R}$ is called nabla differentiable at $t \in \mathbb{T}_{k}$, with nabla derivative $f^{\nabla}(t)$, if there exists $f^{\nabla}(t) \in \mathbb{R}$ such that for any $\epsilon>0$, there exists a neighborhood $V$ of $t$, such that

$$
\left|f(\rho(t))-f(s)-f^{\nabla}(t)(\rho(t)-s)\right| \leq \epsilon|\rho(t)-s|,
$$

for all $s \in V$.

A function $f: \mathbb{T} \rightarrow \mathbb{R}$ is said to be left-dense continuous (ld-continuous), provided it is continuous at all left-dense points in $\mathbb{T}$ and its right-sided limits exist (finite) at all right-dense points in $\mathbb{T}$. The set of all ld-continuous functions is denoted by $C_{l d}(\mathbb{T}, \mathbb{R})$.

The next definition is given in $[3,7,8]$.

Definition 2. A function $G: \mathbb{T} \rightarrow \mathbb{R}$ is called a nabla antiderivative of $g: \mathbb{T} \rightarrow \mathbb{R}$, provided that $G^{\nabla}(t)=g(t)$ holds for all $t \in \mathbb{T}_{k}$. Then the nabla integral of $g$ is defined by

$$
\int_{a}^{b} g(t) \nabla t=G(b)-G(a)
$$

Now we present short introduction of diamond- $\alpha$ derivative as given in $[1,19]$.

Let $\mathbb{T}$ be a time scale and $f(t)$ be differentiable on $\mathbb{T}$ in the $\Delta$ and $\nabla$ senses. For $t \in \mathbb{T}_{k}^{k}$, where $\mathbb{T}_{k}^{k}=\mathbb{T}^{k} \cap \mathbb{T}_{k}$, the diamond- $\alpha$ dynamic derivative $f^{\diamond_{\alpha}}(t)$ is defined by

$$
f^{\diamond_{\alpha}}(t)=\alpha f^{\Delta}(t)+(1-\alpha) f^{\nabla}(t), \quad 0 \leq \alpha \leq 1 .
$$

Thus $f$ is diamond- $\alpha$ differentiable if and only if $f$ is $\Delta$ and $\nabla$ differentiable.

The diamond- $\alpha$ derivative reduces to the standard $\Delta$-derivative for $\alpha=1$, or the standard $\nabla$-derivative for $\alpha=0$. It represents a weighted dynamic derivative for $\alpha \in(0,1)$.

Theorem 4. [19] Let $f, g: \mathbb{T} \rightarrow \mathbb{R}$ be diamond- $\alpha$ differentiable at $t \in \mathbb{T}$ and we write $f^{\sigma}(t)=f(\sigma(t)), g^{\sigma}(t)=g(\sigma(t)), f^{\rho}(t)=f(\rho(t))$ and $g^{\rho}(t)=g(\rho(t))$. Then

(i) $f \pm g: \mathbb{T} \rightarrow \mathbb{R}$ is diamond- $\alpha$ differentiable at $t \in \mathbb{T}$, with

$$
(f \pm g)^{\diamond_{\alpha}}(t)=f^{\diamond_{\alpha}}(t) \pm g^{\diamond_{\alpha}}(t) .
$$

(ii) $f g: \mathbb{T} \rightarrow \mathbb{R}$ is diamond- $\alpha$ differentiable at $t \in \mathbb{T}$, with

$$
(f g)^{\diamond_{\alpha}}(t)=f^{\diamond_{\alpha}}(t) g(t)+\alpha f^{\sigma}(t) g^{\Delta}(t)+(1-\alpha) f^{\rho}(t) g^{\nabla}(t) .
$$

(iii) For $g(t) g^{\sigma}(t) g^{\rho}(t) \neq 0, \frac{f}{g}: \mathbb{T} \rightarrow \mathbb{R}$ is diamond- $\alpha$ differentiable at $t \in \mathbb{T}$, with

$$
\left(\frac{f}{g}\right)^{\diamond_{\alpha}}(t)=\frac{f^{\diamond_{\alpha}}(t) g^{\sigma}(t) g^{\rho}(t)-\alpha f^{\sigma}(t) g^{\rho}(t) g^{\Delta}(t)-(1-\alpha) f^{\rho}(t) g^{\sigma}(t) g^{\nabla}(t)}{g(t) g^{\sigma}(t) g^{\rho}(t)} .
$$


Definition 3. [19] Let $a, t \in \mathbb{T}$ and $h: \mathbb{T} \rightarrow \mathbb{R}$. Then the diamond- $\alpha$ integral from a to $t$ of $h$ is defined by

$$
\int_{a}^{t} h(s) \diamond_{\alpha} s=\alpha \int_{a}^{t} h(s) \Delta s+(1-\alpha) \int_{a}^{t} h(s) \nabla s, \quad 0 \leq \alpha \leq 1,
$$

provided that there exist delta and nabla integrals of $h$ on $\mathbb{T}$.

Theorem 5. [19] Let $a, b, t \in \mathbb{T}, c \in \mathbb{R}$. Assume that $f(s)$ and $g(s)$ are $\diamond_{\alpha}$-integrable functions on $[a, b]_{\mathbb{T}}$. Then

(i) $\int_{a}^{t}[f(s) \pm g(s)] \diamond_{\alpha} s=\int_{a}^{t} f(s) \diamond_{\alpha} s \pm \int_{a}^{t} g(s) \diamond_{\alpha} s ;$

(ii) $\int_{a}^{t} c f(s) \diamond_{\alpha} s=c \int_{a}^{t} f(s) \diamond_{\alpha} s$;

(iii) $\int_{a}^{t} f(s) \diamond_{\alpha} s=-\int_{t}^{a} f(s) \diamond_{\alpha} s$;

(iv) $\int_{a}^{t} f(s) \diamond_{\alpha} s=\int_{a}^{b} f(s) \diamond_{\alpha} s+\int_{b}^{t} f(s) \diamond_{\alpha} s$

(v) $\int_{a}^{a} f(s) \diamond_{\alpha} s=0$.

We need the following results.

Definition 4. [9] A function $f: \mathbb{T} \rightarrow \mathbb{R}$ is called convex on $I_{\mathbb{T}}=I \cap \mathbb{T}$, where $I$ is an interval of $\mathbb{R}$ (open or closed), if

$$
f(\lambda t+(1-\lambda) s) \leq \lambda f(t)+(1-\lambda) f(s),
$$

for all $t, s \in I_{\mathbb{T}}$ and all $\lambda \in[0,1]$ such that $\lambda t+(1-\lambda) s \in I_{\mathbb{T}}$.

The function $f$ is strictly convex on $I_{\mathbb{T}}$ if the inequality (2.1) is strict for distinct $t, s \in I_{\mathbb{T}}$ and $\lambda \in(0,1)$.

The function $f$ is concave (respectively, strictly concave) on $I_{\mathbb{T}}$, if $-f$ is convex (respectively, strictly convex).

Theorem 6. [1] Let $a, b \in \mathbb{T}$ and $c, d \in \mathbb{R}$. Suppose that $g \in C\left([a, b]_{\mathbb{T}},(c, d)\right)$ and $h \in$ $C\left([a, b]_{\mathbb{T}}, \mathbb{R}\right)$ with $\int_{a}^{b}|h(s)| \diamond_{\alpha} s>0$. If $\Phi \in C((c, d), \mathbb{R})$ is convex, then generalized Jensen's Inequality is

$$
\Phi\left(\frac{\int_{a}^{b}|h(s)| g(s) \diamond_{\alpha} s}{\int_{a}^{b}|h(s)| \diamond_{\alpha} s}\right) \leq \frac{\int_{a}^{b}|h(s)| \Phi(g(s)) \diamond_{\alpha} s}{\int_{a}^{b}|h(s)| \diamond_{\alpha} s} .
$$

If $\Phi$ is strictly convex, then the inequality $\leq$ can be replaced by $<$.

Example 1. [1] One of the three most popular examples of calculus on time scales is quantum calculus, i. e., $q^{\mathbb{N}_{0}}=\left\{q^{n}: n \in \mathbb{N}_{0}\right\}$, where $\mathbb{N}_{0}$ is the set of nonnegative integers.

If we set $\mathbb{T}=q^{\mathbb{N}_{0}}$ for $q>1$ and $m<n$, then

$$
\int_{q^{m}}^{q^{n}} f(x) \diamond_{\alpha} x=(q-1) \sum_{i=m}^{n-1} q^{i}\left[\alpha f\left(q^{i}\right)+(1-\alpha) f\left(q^{i+1}\right)\right]
$$

for $m, n \in \mathbb{N}_{0}$. 


\section{Main Results}

In order to present our main results, first we present an extension of Radon's Inequality via time scales.

Theorem 7. Let $w, f, g \in C\left([a, b]_{\mathbb{T}}, \mathbb{R}\right)$ be $\diamond_{\alpha}$-integrable functions, where $w(x), g(x) \neq 0$, $\forall x \in[a, b]_{\mathbb{T}}$. If $\beta \geq \gamma \geq 0$, then

$$
\left(\int_{a}^{b}|w(x)| \diamond_{\alpha} x\right)^{\gamma-\beta} \frac{\left(\int_{a}^{b}|w(x)||f(x)| \diamond_{\alpha} x\right)^{\beta+1}}{\left(\int_{a}^{b}|w(x)||g(x)| \diamond_{\alpha} x\right)^{\gamma}} \leq \int_{a}^{b} \frac{|w(x)||f(x)|^{\beta+1}}{|g(x)|^{\gamma}} \diamond_{\alpha} x .
$$

Equality holds in (3.1), when $f(x) \equiv g(x) \equiv c$, where $c$ is a nonzero real constant.

P r o o f. The right hand side of (3.1) can be written as

$$
\int_{a}^{b} \frac{|w(x)||f(x)|^{\beta+1}}{|g(x)|^{\gamma}} \diamond_{\alpha} x=\int_{a}^{b}|w(x)||g(x)| \diamond_{\alpha} x \times \int_{a}^{b} \frac{|w(x)||g(x)|(\Psi(x))^{\gamma+1}}{\int_{a}^{b}|w(x)||g(x)| \diamond_{\alpha} x} \diamond_{\alpha} x,
$$

where

$$
\Psi(x)=\frac{|f(x)|^{\frac{\beta+1}{\gamma+1}}}{|g(x)|} .
$$

The function $\Phi:[0, \infty) \rightarrow[0, \infty)$ defined by $\Phi(x)=x^{\gamma+1}$ is convex for $x \in[0, \infty)$, so applying generalized Jensen's Inequality given in (2.2), we have

$$
\left(\int_{a}^{b} \frac{|w(x)||g(x)| \Psi(x)}{\int_{a}^{b}|w(x)||g(x)| \diamond_{\alpha} x} \diamond_{\alpha} x\right)^{\gamma+1} \leq \int_{a}^{b} \frac{|w(x)||g(x)|(\Psi(x))^{\gamma+1}}{\int_{a}^{b}|w(x)||g(x)| \diamond_{\alpha} x} \diamond_{\alpha} x .
$$

Then (3.3) can be written as

$$
\left(\int_{a}^{b} \frac{|w(x)||f(x)|^{\frac{\beta+1}{\gamma+1}}}{\int_{a}^{b}|w(x)||g(x)| \diamond_{\alpha} x} \diamond_{\alpha} x\right)^{\gamma+1} \leq \int_{a}^{b} \frac{|w(x)||g(x)| \frac{|f(x)|^{\beta+1}}{|g(x)|^{\gamma+1}}}{\int_{a}^{b}|w(x)||g(x)| \diamond_{\alpha} x} \diamond_{\alpha} x .
$$

Now the function $\Phi:[0, \infty) \rightarrow[0, \infty)$ defined by $\Phi(x)=x^{\frac{\beta+1}{\gamma+1}}$ is convex for $x \in[0, \infty)$, so applying generalized Jensen's Inequality, we have

$$
\left(\int_{a}^{b} \frac{|w(x)||f(x)| \diamond_{\alpha} x}{\int_{a}^{b}|w(x)| \diamond_{\alpha} x}\right)^{\frac{\beta+1}{\gamma+1}} \leq \int_{a}^{b} \frac{|w(x)||f(x)|^{\frac{\beta+1}{\gamma+1}} \diamond_{\alpha} x}{\int_{a}^{b}|w(x)| \diamond_{\alpha} x}
$$

Now (3.5) becomes

$$
\left(\int_{a}^{b}|w(x)| \diamond_{\alpha} x\right)^{\frac{\gamma-\beta}{\gamma+1}}\left(\int_{a}^{b}|w(x)||f(x)| \diamond_{\alpha} x\right)^{\frac{\beta+1}{\gamma+1}} \leq \int_{a}^{b}|w(x)||f(x)|^{\frac{\beta+1}{\gamma+1}} \diamond_{\alpha} x .
$$

From (3.2), (3.4) and (3.6), we get (3.1).

Clearly equality holds in (3.1), when $f(x) \equiv g(x) \equiv c$, where $c$ is a nonzero real constant, which completes the proof. 
Remark 1. If we set $\alpha=1, \mathbb{T}=\mathbb{Z}, w(x)=1, \beta=\gamma=1, f(k)=x_{k} \in \mathbb{R}$ and $g(k)=y_{k} \in(0, \infty)$ for $k \in\{1,2, \ldots, n\}, n \in \mathbb{N}$, then (3.1) reduces to (1.1).

Remark 2. If we set $\alpha=1, \mathbb{T}=\mathbb{Z}, w(x)=1, \beta=\gamma \geq 0, f(k)=x_{k} \in[0, \infty)$ and $g(k)=y_{k} \in$ $(0, \infty)$ for $k \in\{1,2, \ldots, n\}, n \in \mathbb{N}$, then (3.1) reduces to (1.2).

Remark 3. If we set $\alpha=1, \mathbb{T}=\mathbb{Z}, w(x)=1, \beta \geq \gamma \geq 0, f(k)=x_{k} \in[0, \infty)$ and $g(k)=y_{k} \in$ $(0, \infty)$ for $k \in\{1,2, \ldots, n\}, n \in \mathbb{N}$, then discrete version of (3.1) reduces to (1.3).

Example 2. When $\mathbb{T}=\mathbb{R}$, then continuous version of (3.1) can be written as

$$
\left(\int_{a}^{b}|w(x)| d x\right)^{\gamma-\beta} \frac{\left(\int_{a}^{b}|w(x)||f(x)| d x\right)^{\beta+1}}{\left(\int_{a}^{b}|w(x)||g(x)| d x\right)^{\gamma}} \leq \int_{a}^{b} \frac{|w(x)||f(x)|^{\beta+1}}{|g(x)|^{\gamma}} d x .
$$

If we set $[a, b]_{\mathbb{T}}=\left[q^{m}, q^{n}\right]_{q^{\mathbb{N}_{0}}}$ for $q>1$ and $m<n$, where $m, n \in \mathbb{N}_{0}$ and $\mathbb{N}_{0}$ is the set of nonnegative integers, then

$$
\begin{gathered}
{\left[\sum_{i=m}^{n-1} q^{i}\left\{\alpha\left|w\left(q^{i}\right)\right|+(1-\alpha)\left|w\left(q^{i+1}\right)\right|\right\}\right]^{\gamma-\beta} \times \frac{\left[\sum_{i=m}^{n-1} q^{i}\left\{\alpha\left|w\left(q^{i}\right)\right|\left|f\left(q^{i}\right)\right|+(1-\alpha)\left|w\left(q^{i+1}\right)\right|\left|f\left(q^{i+1}\right)\right|\right\}\right]^{\beta+1}}{\left[\sum_{i=m}^{n-1} q^{i}\left\{\alpha\left|w\left(q^{i}\right)\right|\left|g\left(q^{i}\right)\right|+(1-\alpha)\left|w\left(q^{i+1}\right)\right|\left|g\left(q^{i+1}\right)\right|\right\}\right]^{\gamma}}} \\
\leq \sum_{i=m}^{n-1} q^{i}\left\{\alpha \frac{\left|w\left(q^{i}\right)\right|\left|f\left(q^{i}\right)\right|^{\beta+1}}{\left|g\left(q^{i}\right)\right|^{\gamma}}+(1-\alpha) \frac{\left|w\left(q^{i+1}\right)\right|\left|f\left(q^{i+1}\right)\right|^{\beta+1}}{\left|g\left(q^{i+1}\right)\right|^{\gamma}}\right\} .
\end{gathered}
$$

Corollary 1. Let $w, f, g \in C\left([a, b]_{\mathbb{T}}, \mathbb{R}-\{0\}\right)$ be $\diamond_{\alpha}$-integrable functions. If $\gamma \leq \beta \leq-1$, then

$$
\left(\int_{a}^{b}|w(x)| \diamond_{\alpha} x\right)^{\gamma-\beta} \frac{\left(\int_{a}^{b}|w(x)||f(x)| \diamond_{\alpha} x\right)^{\beta+1}}{\left(\int_{a}^{b}|w(x)||g(x)| \diamond_{\alpha} x\right)^{\gamma}} \leq \int_{a}^{b} \frac{|w(x)||f(x)|^{\beta+1}}{|g(x)|^{\gamma}} \diamond_{\alpha} x .
$$

Equality holds in (3.7), when $f(x) \equiv g(x) \equiv c$, where $c$ is a nonzero real constant.

$\mathrm{P}$ r o o f. For $\beta \leq-1, \gamma \leq-1$, the inequalities $-\gamma \geq-\beta,-\gamma \geq 1,-\beta \geq 1$ hold. Taking into account inequality (3.1), we obtain

$$
\begin{aligned}
& \int_{a}^{b} \frac{|w(x)||f(x)|^{\beta+1}}{|g(x)|^{\gamma}} \diamond_{\alpha} x=\int_{a}^{b} \frac{|w(x)||g(x)|^{-\gamma}}{|f(x)|^{-\beta-1}} \diamond_{\alpha} x \\
\geq & \left(\int_{a}^{b}|w(x)| \diamond_{\alpha} x\right)^{-\beta+\gamma} \frac{\left(\int_{a}^{b}|w(x)||g(x)| \diamond_{\alpha} x\right)^{-\gamma}}{\left(\int_{a}^{b}|w(x)||f(x)| \diamond_{\alpha} x\right)^{-\beta-1}} \\
= & \left(\int_{a}^{b}|w(x)| \diamond_{\alpha} x\right)^{\gamma-\beta} \frac{\left(\int_{a}^{b}|w(x)||f(x)| \diamond_{\alpha} x\right)^{\beta+1}}{\left(\int_{a}^{b}|w(x)||g(x)| \diamond_{\alpha} x\right)^{\gamma}}
\end{aligned}
$$

thus inequality (3.7) holds. Clearly the equality holds in (3.7), when $f(x) \equiv g(x) \equiv c$, where $c$ is a nonzero real constant. 
Corollary 2. Let $w, f, g \in C\left([a, b]_{\mathbb{T}}, \mathbb{R}\right)$ be $\diamond_{\alpha}$-integrable functions, where $w(x), g(x) \neq 0$, $\forall x \in[a, b]_{\mathbb{T}}$. If $\beta \geq 0$, then

$$
\frac{\left(\int_{a}^{b}|w(x)||f(x)| \diamond_{\alpha} x\right)^{\beta+1}}{\left(\int_{a}^{b}|w(x)||g(x)| \diamond_{\alpha} x\right)^{\beta}} \leq \int_{a}^{b} \frac{|w(x)||f(x)|^{\beta+1}}{|g(x)|^{\beta}} \diamond_{\alpha} x
$$

Equality holds in (3.8), when $f(x)=c g(x)$, where $c$ is a real constant.

$\mathrm{P}$ r o o f. If we put $\beta=\gamma$ in (3.1), then we get (3.8), which is Radon's Inequality on dynamic time scales. Clearly the equality holds in (3.8), if $f(x)=c g(x)$, where $c$ is a real constant.

Corollary 3. Let $w, f, g \in C\left([a, b]_{\mathbb{T}}, \mathbb{R}-\{0\}\right)$ be $\diamond_{\alpha}$-integrable functions. If $\beta \leq-1$, then

$$
\frac{\left(\int_{a}^{b}|w(x)||f(x)| \diamond_{\alpha} x\right)^{\beta+1}}{\left(\int_{a}^{b}|w(x)||g(x)| \diamond_{\alpha} x\right)^{\beta}} \leq \int_{a}^{b} \frac{|w(x)||f(x)|^{\beta+1}}{|g(x)|^{\beta}} \diamond_{\alpha} x .
$$

Equality holds in (3.9), when $f(x)=c g(x)$, where $c$ is a nonzero real constant.

P r o o f. By applying inequality (3.8) for $\beta \leq-1$, we obtain

$$
\int_{a}^{b} \frac{|w(x)||f(x)|^{\beta+1}}{|g(x)|^{\beta}} \diamond_{\alpha} x=\int_{a}^{b} \frac{|w(x)||g(x)|^{-\beta}}{|f(x)|^{-\beta-1}} \diamond_{\alpha} x \geq \frac{\left(\int_{a}^{b}|w(x)||g(x)| \diamond_{\alpha} x\right)^{-\beta}}{\left(\int_{a}^{b}|w(x)||f(x)| \diamond_{\alpha} x\right)^{-\beta-1}}
$$

thus inequality (3.9) holds. Clearly the equality holds in (3.9), if $f(x)=c g(x)$, where $c$ is a nonzero real constant.

Corollary 4. Let $w, f, g \in C\left([a, b]_{\mathbb{T}}, \mathbb{R}-\{0\}\right)$ be $\diamond_{\alpha}$-integrable functions. If $\beta>0$ or $\beta \leq-1$, then

$$
\frac{\left(\int_{a}^{b}|w(x)||f(x)| \diamond_{\alpha} x\right)^{\beta+1}}{\left(\int_{a}^{b}|w(x)||f(x)||g(x)|^{\frac{1}{\beta}} \diamond_{\alpha} x\right)^{\beta}} \leq \int_{a}^{b} \frac{|w(x)||f(x)|}{|g(x)|} \diamond_{\alpha} x .
$$

P r o o f. Replace $|g(x)|$ by $|f(x) \| g(x)|^{\frac{1}{\beta}}$ in (3.8) and (3.9), then inequality (3.10) is obtained.

Corollary 5. Let $w, f \in C\left([a, b]_{\mathbb{T}}, \mathbb{R}\right)$ be $\diamond_{\alpha}$-integrable functions, where $w(x) \neq 0, \forall x \in[a, b]_{\mathbb{T}}$. If $\eta_{2} \geq \eta_{1}>0$, then

$$
\left(\frac{\int_{a}^{b}|w(x)||f(x)|^{\eta_{1}} \diamond_{\alpha} x}{\int_{a}^{b}|w(x)| \diamond_{\alpha} x}\right)^{\frac{1}{\eta_{1}}} \leq\left(\frac{\int_{a}^{b}|w(x)||f(x)|^{\eta_{2}} \diamond_{\alpha} x}{\int_{a}^{b}|w(x)| \diamond_{\alpha} x}\right)^{\frac{1}{\eta_{2}}}
$$


P r o o f. Take $\beta \geq 0,1+\beta=\frac{\eta_{2}}{\eta_{1}} \geq 1$ and $g(x)=1$, then (3.8) becomes

$$
\frac{\left(\int_{a}^{b}|w(x)||f(x)| \diamond_{\alpha} x\right)^{\frac{\eta_{2}}{\eta_{1}}}}{\left(\int_{a}^{b}|w(x)| \diamond_{\alpha} x\right)^{\frac{\eta_{2}}{\eta_{1}}-1}} \leq \int_{a}^{b}|w(x)||f(x)|^{\frac{\eta_{2}}{\eta_{1}}} \diamond_{\alpha} x .
$$

Dividing (3.12) by $\int_{a}^{b}|w(x)| \diamond_{\alpha} x$, replacing $|f(x)|$ by $|f(x)|^{\eta_{1}}$ and taking power $1 / \eta_{2}>0$, we get the inequality (3.11), which is known as the dynamic weighted power mean inequality on time scales.

Remark 4. Set $\beta \geq 0,1+\beta=\eta_{2} / \eta_{1} \geq 1, g(x)=1$ and $\int_{a}^{b}|w(x)| \diamond_{\alpha} x=1$, then (3.11) becomes

$$
\left(\int_{a}^{b}|w(x)||f(x)|^{\eta_{1}} \diamond_{\alpha} x\right)^{\frac{1}{\eta_{1}}} \leq\left(\int_{a}^{b}|w(x)||f(x)|^{\eta_{2}} \diamond_{\alpha} x\right)^{\frac{1}{\eta_{2}}} .
$$

Inequality given in (3.13) is called Schlömilch's Inequality on time scales.

Let $w, f \in C_{r d}\left([a, b]_{\mathbb{T}},[0, \infty)\right)$, then for $\alpha=1$, inequality (3.13) takes the form

$$
\left(\int_{a}^{b} w(x) f^{\eta_{1}}(x) \Delta x\right)^{\frac{1}{\eta_{1}}} \leq\left(\int_{a}^{b} w(x) f^{\eta_{2}}(x) \Delta x\right)^{\frac{1}{\eta_{2}}}
$$

as given in [12, Lemma $\mathrm{A}]$.

Let $w, f \in C\left([a, b]_{\mathbb{T}},[0, \infty)\right)$, then inequality (3.13) takes the form

$$
\left(\int_{a}^{b} w(x) f^{\eta_{1}}(x) \diamond_{\alpha} x\right)^{\frac{1}{\eta_{1}}} \leq\left(\int_{a}^{b} w(x) f^{\eta_{2}}(x) \diamond_{\alpha} x\right)^{\frac{1}{\eta_{2}}},
$$

as given in [20, Lemma 3.4].

Now we present generalized Nesbitt's Inequality on dynamic time scale calculus.

Theorem 8. Let $w, f \in C\left([a, b]_{\mathbb{T}}, \mathbb{R}-\{0\}\right)$ be $\diamond_{\alpha}$-integrable functions, $c, d \in \mathbb{R}$ and

$$
c \int_{a}^{b}|w(x)||f(x)| \diamond_{\alpha} x-d|f(x)|>0
$$

where $x \in[a, b]_{\mathbb{T}}$. If $\beta \geq \gamma \geq 0$, then

$$
\begin{gathered}
\left(\int_{a}^{b}|w(x)| \diamond_{\alpha} x\right)^{2 \gamma-\beta} \frac{\left(\int_{a}^{b}|w(x)||f(x)| \diamond_{\alpha} x\right)^{\beta-2 \gamma+1}}{\left(c \int_{a}^{b}|w(x)| \diamond_{\alpha} x-d\right)^{\gamma}} \\
\leq \int_{a}^{b} \frac{|w(x)||f(x)|^{\beta-\gamma+1}}{\left(c \int_{a}^{b}|w(x)||f(x)| \diamond_{\alpha} x-d|f(x)|\right)^{\gamma} \diamond_{\alpha} x .}
\end{gathered}
$$


P r o o f. By using inequality (3.1), we have that

$$
\begin{gathered}
\int_{a}^{b} \frac{|w(x)||f(x)|^{\beta-\gamma+1}}{\left(c \int_{a}^{b}|w(x)||f(x)| \diamond_{\alpha} x-d|f(x)|\right)^{\gamma} \diamond_{\alpha} x} \\
=\int_{a}^{b} \frac{|w(x)||f(x)|^{\beta+1}}{\left(c|f(x)| \int_{a}^{b}|w(x)||f(x)| \diamond_{\alpha} x-d|f(x)|^{2}\right)^{\gamma} \diamond_{\alpha} x} \\
\geq\left(\int_{a}^{b}|w(x)| \diamond_{\alpha} x\right)^{\gamma-\beta} \frac{\left(\int_{a}^{b}|w(x)||f(x)| \diamond_{\alpha} x\right)^{\beta+1}}{\left[c\left(\int_{a}^{b}|w(x)||f(x)| \diamond_{\alpha} x\right)^{2}-d \int_{a}^{b}|w(x)||f(x)|^{2} \diamond_{\alpha} x\right]^{\gamma}} .
\end{gathered}
$$

Taking Jensen's Inequality into account, we have

$$
\left(\int_{a}^{b} \frac{|w(x)||f(x)| \diamond_{\alpha} x}{\int_{a}^{b}|w(x)| \diamond_{\alpha} x}\right)^{2} \leq \int_{a}^{b} \frac{|w(x)||f(x)|^{2} \diamond_{\alpha} x}{\int_{a}^{b}|w(x)| \diamond_{\alpha} x}
$$

Then, using inequality (3.15), we have that

$$
\begin{gathered}
c\left(\int_{a}^{b}|w(x)||f(x)| \diamond_{\alpha} x\right)^{2}-d \int_{a}^{b}|w(x)||f(x)|^{2} \diamond_{\alpha} x \\
\leq c\left(\int_{a}^{b}|w(x)||f(x)| \diamond_{\alpha} x\right)^{2}-\frac{d}{\int_{a}^{b}|w(x)| \diamond_{\alpha} x}\left(\int_{a}^{b}|w(x)||f(x)| \diamond_{\alpha} x\right)^{2} \\
=\left(\frac{c \int_{a}^{b}|w(x)| \diamond_{\alpha} x-d}{\int_{a}^{b}|w(x)| \diamond_{\alpha} x}\right)\left(\int_{a}^{b}|w(x)||f(x)| \diamond_{\alpha} x\right)^{2} .
\end{gathered}
$$

And then

$$
\begin{gathered}
\left(\int_{a}^{b}|w(x)||f(x)| \diamond_{\alpha} x\right)^{\beta+1} \\
{\left[c\left(\int_{a}^{b}|w(x)||f(x)| \diamond_{\alpha} x\right)^{2}-d \int_{a}^{b}|w(x)||f(x)|^{2} \diamond_{\alpha} x\right]^{\gamma}} \\
\geq\left(\frac{\int_{a}^{b}|w(x)| \diamond_{\alpha} x}{c \int_{a}^{b}|w(x)| \diamond_{\alpha} x-d}\right)^{\gamma}\left(\int_{a}^{b}|w(x)||f(x)| \diamond_{\alpha} x\right)^{\beta-2 \gamma+1}
\end{gathered}
$$


So,

$$
\begin{gathered}
\left(\frac{\int_{a}^{b}|w(x)| \diamond_{\alpha} x}{c \int_{a}^{b}|w(x)| \diamond_{\alpha} x-d}\right)^{\gamma}\left(\int_{a}^{b}|w(x)||f(x)| \diamond_{\alpha} x\right)^{\beta-2 \gamma+1} \\
\leq\left(\int_{a}^{b}|w(x)| \diamond_{\alpha} x\right)^{\beta-\gamma} \int_{a}^{b} \frac{|w(x)||f(x)|^{\beta-\gamma+1}}{\left(c \int_{a}^{b}|w(x)||f(x)| \diamond_{\alpha} x-d|f(x)|\right)^{\gamma}} \diamond_{\alpha} x .
\end{gathered}
$$

Clearly (3.14) holds true from (3.16).

Remark 5. If we set $\alpha=1, \mathbb{T}=\mathbb{Z}, w(x)=1, \beta=\gamma=1$ and $f(k)=x_{k} \in(0, \infty)$ for $k \in\{1,2, \ldots, n\}, n \in \mathbb{N}-\{1\}$, then discrete version of (3.14) reduces to

$$
\frac{n}{c n-d} \leq \sum_{k=1}^{n} \frac{x_{k}}{c X_{n}-d x_{k}}
$$

where $X_{n}=x_{1}+x_{2}+\ldots+x_{n}$.

Inequality (3.17) is called generalized Nesbitt's Inequality as given in [10].

Further if we set $n=3$ and $c=d$, where $c, d \in(0, \infty)$, then (3.17) takes the form

$$
\frac{3}{2} \leq \frac{x_{1}}{x_{2}+x_{3}}+\frac{x_{2}}{x_{3}+x_{1}}+\frac{x_{3}}{x_{1}+x_{2}},
$$

where $x_{1}, x_{2}, x_{3}>0$. Inequality (3.18) is called Nesbitt's Inequality as given in [14].

Remark 6. If we set $\alpha=1$, then we get delta versions of dynamic inequalities and if we set $\alpha=0$, then we get nabla versions of dynamic inequalities presented in this article.

Further if we put $\mathbb{T}=\mathbb{Z}$, then we get discrete versions of dynamic inequalities and if we put $\mathbb{T}=\mathbb{R}$, then we get continuous versions of dynamic inequalities presented in this article.

\section{Conclusion and Future Work}

In this research article, we have presented dynamic inequalities on diamond- $\alpha$ calculus such as Radon's Inequality, Bergström's Inequality, the weighted power mean inequality, Schlömilch's Inequality and Nesbitt's Inequality.

We can generalize dynamic inequalities using functional generalization as given in [20]. We can present dynamic inequalities on fractional calculus as given in [16] and on quantum calculus. We can develop dynamic inequalities using fractional Riemann-Liouville integral on time scale calculus in a similar fashion as given in [2] and [17]. Similarly we can generalize dynamic inequalities of this article using time scales fractional derivative as given in [2]. It will be interesting to present dynamic inequalities in two or more dimensions.

Recently it has found that many dynamic inequalities such as Radon's Inequality, the weighted power mean inequality, Schlömilch's Inequality, Rogers-Hölder's Inequality and Bernoulli's Inequality are equivalent on time scales as given in [18], so we can find more equivalent dynamic inequalities on time scales.

\section{REFERENCES}

1. Agarwal R.P., O'Regan D., Saker S.H. Dynamic Inequalities on Time Scales. Springer International Publishing, Cham, Switzerland, 2014. DOI: 10.1007/978-3-319-11002-8 
2. Anastassiou G. A. Integral operator inequalities on time scales. Intern. J. Difference Equ., 2012. Vol. 7, No. 2. P. 111-137.

3. Anderson D., Bullock J., Erbe L., Peterson A., Tran H. Nabla dynamic equations on time scales. PanAmerican Math. J., 2003. Vol. 13, No. 1. P. 1-48.

4. Beckenbach E.F., Bellman R. Inequalities. Springer, Berlin, Göttingen and Heidelberg, 1961. DOI: $10.1007 / 978-3-642-64971-4$

5. Bellman R. Notes on matrix theory-IV (An inequality due to Bergström). Amer. Math. Monthly, 1955. Vol. 62. P. 172-173.

6. Bergström H. A triangle inequality for matrices. Den Elfte Skandinaviske Matematikerkongress, 1949, Trondheim, Johan Grundt Tanums Forlag, Oslo, 1952. P. 264-267.

7. Bohner M., Peterson A. Dynamic Equations on Time Scales. Birkhäuser Boston, Inc., Boston, MA, 2001. DOI: 10.1007/978-1-4612-0201-1

8. Bohner M., Peterson A. Advances in Dynamic Equations on Time Scales. Birkhäuser Boston, Boston, MA, 2003. DOI: $10.1007 / 978-0-8176-8230-9$

9. Dinu C. Convex functions on time scales. Annals Univ. of Craiova, Math. Comp. Sci. Ser., 2008. Vol. 35. P. 87-96.

10. Bătineţu-Giurgiu D. M., Mărghidanu D., Pop O.T. A new generalization of Radon's Inequality and applications. Creative Math \& Inf, 2011. Vol. 20, No. 2. P. 111-116.

11. Hilger S. Ein Maßkettenkalkül mit Anwendung auf Zentrumsmannigfaltigkeiten. PhD thesis, Universität Würzburg, 1988.

12. Hong C. H., Yeh C. C. Rogers-Hölder's Inequality on time scales. Intern. J. Pure Appl. Math., 2006. Vol. 29, No. 3. P. 289-309.

13. Mitrinović D. S. Analytic Inequalities. Springer-Verlag, Berlin, 1970. DOI: 10.1007/978-3-642-99970-3

14. Nesbitt A. M. Problem 15114. Educational Times, 1903. Vol. 3. P. 37-38.

15. Radon J. Theorie und Anwendungen der absolut additiven Mengenfunktionen. Sitzungsber. Acad. Wissen. Wien, 1913. Vol. 122. P. 1295-1438.

16. Sahir M. J. S. Dynamic inequalities for convex functions harmonized on time scales. J. Appl. Math. Phys., 2017. Vol. 5. P. 2360-2370. DOI: 10.4236/jamp.2017.512193

17. Sahir M. J.S. Fractional dynamic inequalities harmonized on time scales. Cogent Math. Stat., 2018. Vol. 5. P. 1-7. DOI: 10.1080/23311835.2018.1438030

18. Sahir M. J. S. Hybridization of classical inequalities with equivalent dynamic inequalities on time scale calculus. The Teaching of Mathematics, 2018. Vol. 21, No. 1. P. 38-52.

19. Sheng Q., Fadag M., Henderson J., Davis J. M. An exploration of combined dynamic derivatives on time scales and their applications. Nonlinear Anal. Real World Appl., 2006. Vol. 7, No. 3. P. $395-413$. DOI: 10.1016/j.nonrwa.2005.03.008

20. Tian J. F. Ha M. H. Extensions of Hölder-type inequalities on time scales and their applications. J. Nonlinear Sci. Appl., 2017. Vol. 10, No. 3. P. 937-953. DOI: 10.22436/jnsa.010.03.07 